\title{
SMOOTHING PROPERTIES OF IMPLICIT FINITE DIFFERENCE METHODS FOR A DIFFUSION EQUATION IN MAXIMUM NORM *
}

\author{
J. THOMAS BEALE $\dagger$
}

\begin{abstract}
We prove a regularity property of finite difference schemes for the heat or diffusion equation $u_{t}=\Delta u$ in maximum norm with large time steps. For a class of time discretizations including $L$-stable single-step methods and the second-order backward difference formula, with the usual second-order Laplacian, we show that solutions of the scheme gain first spatial differences boundedly, and also second differences except for logarithmic factors, with respect to nonhomogeneous terms. A weaker property is shown for the Crank-Nicolson method. As a consequence we show that the numerical solution of a convection-diffusion equation with an interface can allow $O(h)$ truncation error near the interface and still have a solution with uniform $O\left(h^{2}\right)$ accuracy and first differences of uniform accuracy almost $O\left(h^{2}\right)$.
\end{abstract}

Key words. finite difference methods, parabolic equations, diffusion, regularity, stability, Lstable, A-stable, maximum norm, interfaces, moving boundaries, immersed interface method

AMS subject classifications. 65M12,35K05,35R05

1. Introduction. We are concerned with finite difference methods for a diffusion equation in $\mathbb{R}^{d} \times[0, \infty)$ such as

$$
u_{t}=\Delta u+f
$$

which allow large time steps and gain regularity in maximum norm; that is, norms of spatial differences of the discrete solution can be estimated by the norm of $f$. We suppose that, at each time $t, f$ and $u$ are defined on a grid $\mathbb{R}_{h}^{d}=\left\{j h: j \in \mathbb{Z}^{d}\right\}$ in all $d$-space; a rectangular domain with periodic or homogeneous Dirichlet or Neumann boundary conditions can be regarded as a special case. We replace $\Delta$ by the usual second-order discrete Laplacian

$$
\Delta_{h}=\sum_{\nu=1}^{d} D_{\nu}^{+} D_{\nu}^{-}
$$

where $D_{\nu}^{ \pm}$are the forward and backward divided difference operators in direction $\nu$. If the time discretization is a single-step method, with time step $k$, the approximation $u^{n}$ to $u(\cdot, n k)$ in (1.1) with $f=0$ has the form

$$
u^{n}=s\left(k \Delta_{h}\right)^{n} u^{0}
$$

where $s$ is the function so that $s(k \lambda)$ is the corresponding factor for the model equation $y_{t}=\lambda y$. The regularity results proved here include single-step methods that are $L$ stable; that is, $|s(z)| \leq 1$ for all $z \in \mathbb{C}$ with $\operatorname{Re} z \leq 0$ and $s(\infty)=0$. The simplest example is the backward Euler method, but the results are more useful for secondorder methods. Two examples are a modification of the Crank-Nicolson method due to Twizell et al. [25], adapted in [18], which we will call TGA, and a second-order singly diagonally implicit Runge-Kutta method (SDIRK2). (Formulas are given in the Appendix.) The results also apply to multistep methods such as the second-order

\footnotetext{
* This work was supported in part by National Science Foundation grant DMS-0806482.

$\dagger$ Department of Mathematics, Duke University, Box 90320, North Carolina 27708-0320.
} 
backward difference formula (BDF2). These three methods and Crank-Nicolson (CN) have all been useful in applications $([5,6,11,15,18,24,27])$. We show that $\mathrm{CN}$ has a regularity property for the nonhomogeneous equation which is weaker than that for the $L$-stable methods.

The regularity results proved here can be used to estimate the error in the computed solution with limited information about the truncation error. In problems with discontinuities at interfaces, it is desirable to use an ordinary rectangular grid and to allow the truncation error to be $O(h)$ near the interface while $O\left(h^{2}\right)$ elsewhere. Nevertheless, it is often expected in practice that the solution obtained is uniformly second-order accurate. The $O(h)$ truncation error near the interface can be achieved using the immersed interface method of R. LeVeque and Z. Li $[12,14,13,11,15]$ or the related method of A. Mayo [17]; corrections are made to the difference approximations based on jump conditions at the interface. For a Poisson problem it was proved in [3] that the solution obtained in this way is uniformly $O\left(h^{2}\right)$; see also [14, 21]. We prove here a result of the same kind for a linear convection-diffusion equation with a moving interface. This conclusion provides assurance that the expected gain in accuracy in a time-dependent interface problem can be achieved in some circumstances.

We now state the regularity property for single-step methods. The method is called $A(\theta)$-stable if $|s(z)| \leq 1$ on a sector

$$
\Sigma(\theta)=\{z \in \mathbb{C}:|\arg z-\pi| \leq \theta\} \cup\{0\} .
$$

For a linear operator $E$ on $L^{\infty}\left(\mathbb{R}_{h}^{d}\right)$ we use the norm

$$
\|E\|=\sup \|E f\|_{L^{\infty}\left(\mathbb{R}_{h}^{d}\right)} /\|f\|_{L^{\infty}\left(\mathbb{R}_{h}^{d}\right)}, \quad f \neq 0 .
$$

We let $D_{h}$ denote any divided difference operator $D_{\nu}^{ \pm}$and $D_{h}^{2}$ any product of two such operators. We first give bounds for the norm of $s\left(k \Delta_{h}\right)^{n}$ and its differences, and then estimates for the nonhomogeneous equation.

THEOREM 1.1. Suppose the solution of the semi-discrete initial value problem

$$
u_{t}=\Delta_{h} u, \quad u(0)=u^{0}
$$

is approximated by (1.3). Suppose (1.3) is consistent; $s$ is analytic on a sector $\Sigma(\theta)$ for some $\theta>0$ and on a neighborhood of infinity; $s$ is $A(\theta)$-stable; and $s(\infty)=0$. Then for any $h, k$ with $0<h, k \leq 1$ and integer $n>0$,

$$
\begin{gathered}
\left\|s\left(k \Delta_{h}\right)^{n}\right\| \leq C_{0}, \\
\left\|D_{h} s\left(k \Delta_{h}\right)^{n}\right\| \leq C_{1}(n k)^{-1 / 2}, \\
\left\|D_{h}^{2} s\left(k \Delta_{h}\right)^{n}\right\| \leq C_{2}(n k)^{-1}(1+|\log h|+|\log n k|),
\end{gathered}
$$

with operator norm as in (1.5) and constants independent of $h, k$ and $n$.

THEOREM 1.2. With s as above, suppose the problem

$$
u_{t}=\Delta_{h} u+f, \quad u(0)=0
$$

is approximated by

$$
u^{n+1}=s\left(k \Delta_{h}\right) u^{n}+k \sum_{i=1}^{m} q_{i}\left(k \Delta_{h}\right)\left(1-\eta_{i} k \Delta_{h}\right)^{-1} f\left(\cdot, n k+\tau_{i} k\right)
$$


where $k=$ ch for some $c>0 ; m \geq 1, \eta_{i}>0$, and $\tau_{i}$ are fixed numbers; and $q_{i}$ is an analytic function on $\Sigma(\theta)$ such that $q_{i}\left(k \Delta_{h}\right)$ is bounded in norm in $L^{\infty}\left(\mathbb{R}_{h}^{d}\right)$, independently of $h, k$. Then for $0<n k \leq T$,

$$
\begin{gathered}
\left\|u^{n}\right\|+\left\|D_{h} u^{n}\right\| \leq C_{1} \sup _{t \leq T}\|f(\cdot, t)\|, \\
\left\|D_{h}^{2} u^{n}\right\| \leq C_{2}\left(1+|\log h|^{2}\right) \sup _{t \leq T}\|f(\cdot, t)\|,
\end{gathered}
$$

with norms in $L^{\infty}\left(\mathbb{R}_{h}^{d}\right)$ and constants depending on $c$ and $T$ but not on $h$ or $n$.

The present theorems allow regularity with large $k$ by making strong stability assumptions on the choice of time discretization. They apply directly to TGA and SDIRK2 among others, as explained at the end of Sec. 5. Theorem 4.1, proved in Sec. 4, contains Theorem 1.1 and also applies to multistep methods including BDF2. For CN the estimate (1.7) is included in [19], but (1.8), (1.9) do not hold. Surprisingly, (1.12) holds for CN, although (1.13) does not; this is shown in Sec. 7.

If an exact, smooth solution of (1.10) is approximated by (1.11) and has truncation error $O\left(k \cdot\left(h^{2}+k^{2}\right)\right)$ in $L^{\infty}$, with $k=c h$, then Theorem 1.2 applied to the error equation gives $O\left(h^{2}\right)$ convergence for $u$ and $D u$, and $O\left(h^{2}(\log h)^{2}\right)$ for $D^{2} u$, bounded by the maximum norm of the truncation error. To illustrate further the significance of Theorem 1.2, we derive estimates in Sec. 8 for a convection-diffusion equation with an interface. We suppose the equation is posed in a periodic domain with a prescribed moving interface at which there may be a discontinuity. Once a scheme is chosen, the corrections described above leave a truncation error which is $O(h)$ near the interface and $O\left(h^{2}\right)$ elsewhere. Estimates for the case of CN show that for a piecewise smooth solution the error is uniformly $O\left(h^{2}\right)$. If one of the L-stable methods is used, the same is true, and furthermore the error in first differences is $O\left(h^{2}(\log h)^{2}\right)$; a value for the first derivatives of the same accuracy can then be found. The proof depends on the fact that the local $O(h)$ truncation error can be written as a discrete divergence of an $O\left(h^{2}\right)$ function (Lemma 8.1). In [18] a distinction in accuracy and stability was observed between TGA and CN in a finite-volume discretization of a moving boundary problem.

For schemes with $k=c h^{2}$, regularity results like Theorem 1.1 have been proved for general parabolic equations and without logarithmic factors in [26]. The estimate (1.7) was proved in [19] without restriction on $k$ for rational $s$ with weaker assumptions. A smoothing property for CN was proved in [16] in $L^{2}$ norms with initial backward Euler steps, in analogy to (7.2), the weakened version of (1.8) shown here for CN. The technique of proof for Theorems 1.1 and 4.1 is close to that of $[1,22,23]$ and related work, relying on the point of view of analytic semigroups of operators, although the sequence of steps here is different. Resolvent estimates were proved for a general class of discrete elliptic operators in [1], Sec. 4.1, which include (2.26), (2.27) here and appear to imply (2.28); we give a more direct proof for the special case of $\Delta_{h}$. Resolvent estimates like (2.26) have been proved in the finite element setting for general domains; see [2]. For this and smoothing properties with finite elements, see [23].

In Sec. 2 we derive estimates (Thm. 2.1) for the solution operators $e^{\Delta_{h} t}$ of (1.6) and their spatial differences, for complex $t$. These lead to estimates (Thm. 2.2) for the resolvent $\left(z-\Delta_{h}\right)^{-1}$ and its differences for $z \in \mathbb{C}$ outside a sector about the negative axis. For periodic grid functions we give estimates for $\left(\Delta_{h}\right)^{-1}$ and its differences in Sec. 3. The resolvent estimates in Secs. 2 and 3 are close to best possible; there are logarithmic factors for the second differences. In Sec. 4 we prove Theorem 4.1, the 
generalization of Theorem 1.1, by writing the time step operators as contour integrals and estimating, as was done in $[1,23]$. In Sec. 5 we show that Thm. 1.1 follows from Thm. 4.1, prove Thm. 1.2, and comment on TGA and SDIRK2. In Sec. 6 we verify for BDF2 that Thm 4.1 applies and Thm. 1.2 holds. In Sec. 7, we show that (1.12) of Thm. 2.1 holds for CN. Sec. 8 explains the application to the interface problem and verifies the statements above.

2. The exponential and resolvent of the discrete Laplacian. The solution of the semi-discrete initial value problem (1.6) is $u(\cdot, t)=e^{\Delta_{h} t} u^{0}$. We derive estimates for the solution operator $e^{\Delta_{h} t}$ on $L^{\infty}\left(\mathbb{R}_{h}^{d}\right)$ for complex $t$ in a sector about $t>0$. As noted in [23], p. 83, the maximum principle holds for $t>0$, since it holds for the backward Euler approximation (see [20], p. 48). Here we obtain more general estimates by adapting an argument of [10], as presented in [22], for real, discrete time with step $O\left(h^{2}\right)$. We then write the resolvent $\left(z-\Delta_{h}\right)^{-1}$ as an integral of $e^{\Delta_{h} t}$ and estimate its differences for $z$ in the complement of a sector of the form (1.4).

TheOREM 2.1. For any $M>0$ the operator $e^{\Delta_{h} t}$ on $L^{\infty}\left(\mathbb{R}_{h}^{d}\right)$ extends to an analytic function on the sector

$$
\mathbb{T}_{M}=\left\{t=t_{1}+i t_{2}: t_{1}>0,\left|t_{2}\right| \leq M t_{1}\right\}
$$

and has the estimates, with constants depending on $M$ but not on $h$,

$$
\left\|D_{h}^{\alpha} e^{\Delta_{h} t}\right\| \leq C_{m}|t|^{-m / 2}, \quad|\alpha|=m, \quad m \geq 0 .
$$

Here $D_{h}^{\alpha}$ is any mth-order partial difference, where each first-order difference has the form $D_{\nu}^{ \pm}, 1 \leq \nu \leq d$.

Proof. We express the solution of the equation in terms of the discrete Green's function $g(x, t)$ satisfying

$$
g_{t}=\Delta_{h} g, \quad g(j h, 0)=\delta_{j 0},
$$

that is, $g(0,0)=1$ and $g(j h, 0)=0$ for all $j \neq 0$ in $\mathbb{Z}^{d}$. The solution of (1.6) is

$$
u(j h, t)=\sum_{\ell \in \mathbb{Z}^{d}} g(j h-\ell h, t) u^{0}(\ell h) .
$$

Thus for each $j \in \mathbb{Z}^{d}$,

$$
|u(j h, t)| \leq \sum_{j}|g(j h, t)|\left\|u^{0}\right\|_{\infty},
$$

and for the case $m=0$ it will be enough to show that $g(\cdot, t)$ extends analytically in $t$ with

$$
\sum_{j \in \mathbb{Z}^{d}}|g(j h, t)| \leq C_{0}, \quad t \in \mathbb{T}_{M}
$$

Now if $g^{(d)}$ denotes the Green's function in dimension $d$, we have

$$
g^{(d)}\left(j_{1} h, j_{2} h, \ldots, j_{d} h\right)=g^{(1)}\left(j_{1} h\right) g^{(1)}\left(j_{2} h\right) \cdots g^{(1)}\left(j_{d} h\right) .
$$

Thus if we prove (2.6) for dimension $d=1$, it then follows for general $d$. A similar remark applies to differences of $g^{(d)}$. 
We write the discrete Fourier transform of $g$ and its inverse for $d=1$ as

$$
\begin{gathered}
\hat{g}(\xi, t)=\sum_{j \in \mathbb{Z}} g(j h, t) e^{-i j \xi}, \\
g(j h, t)=\frac{1}{2 \pi} \int_{-\pi}^{\pi} \hat{g}(\xi, t) e^{i j \xi} d \xi .
\end{gathered}
$$

In the transform $\Delta_{h}=D^{+} D^{-}$has symbol

$$
\hat{\Delta_{h}}(\xi)=-\frac{4}{h^{2}} \sin ^{2}(\xi / 2)=-\frac{1}{h^{2}} \sigma(\xi), \quad \sigma(\xi) \equiv 4 \sin ^{2}(\xi / 2)
$$

so that (2.3) becomes

$$
\hat{g}_{t}=-\left(\sigma / h^{2}\right) \hat{g}, \quad \hat{g}(\xi, 0)=1
$$

and thus

$$
\hat{g}(\xi, t)=e^{-\sigma(\xi) t / h^{2}}
$$

It will be important that

$$
|\xi| / \pi \leq|\sin (\xi / 2)| \leq|\xi| / 2, \quad|\xi| \leq \pi
$$

so that $\sigma /|\xi|$ is bounded above and below. Formula (2.12) extends analytically to $t=t_{1}+i t_{2} \in \mathbb{C}$, and for $t$ in the sector $\mathbb{T}_{M}$ we have

$$
|\hat{g}(\xi, t)| \leq e^{-\xi^{2} t_{1} /(\pi h)^{2}} \leq e^{-c \xi^{2}|t| / h^{2}},
$$

with $c$ depending on $M$. We will also need the bound

$$
\left|\left(\frac{\partial}{\partial \xi}\right)^{2} \hat{g}(\xi, t)\right| \leq C \frac{|t|}{h^{2}}\left(1+\frac{\xi^{2}|t|}{h^{2}}\right) e^{-c \xi^{2}|t| / h^{2}} .
$$

We now estimate $g(j h, t)$ using $(2.9),(2.14),(2.15)$. The change of variables $\xi=$ $s h / \sqrt{|t|}$ gives

$$
|g(j h, t)| \leq \frac{1}{2 \pi} \int_{-\pi}^{\pi}|\hat{g}(\xi, t)| d \xi \leq \int_{-\infty}^{\infty} e^{-c s^{2}} \frac{h}{\sqrt{|t|}} d s=C \frac{h}{\sqrt{|t|}}
$$

On the other hand, if we integrate by parts twice in (2.9), apply (2.15), and change variables again, we get, for $j \neq 0$,

$$
|g(j h, t)| \leq \frac{C}{j^{2}} \int_{-\pi}^{\pi}\left|\hat{g}_{\xi \xi}(\xi, t)\right| d \xi \leq \frac{C^{\prime}}{j^{2}} \frac{|t|}{h^{2}} \frac{h}{\sqrt{|t|}}=\frac{C^{\prime \prime}}{j^{2}} \frac{\sqrt{|t|}}{h} .
$$

Finally we sum over $j$. Let $J=[\sqrt{|t|} / h]$. For $0<|j| \leq J$ we estimate $|g(j h, t)|$ by (2.16) and for $|j|>J$ we use (2.17), and noting that $|g(0, t)| \leq 1$, we verify (2.6):

$$
\sum_{j \in \mathbb{Z}}|g(j h, t)| \leq 1+C_{1} J(h / \sqrt{|t|})+C_{2}(J+1)^{-1} \sqrt{|t|} / h \leq C .
$$


This proves (2.6) with $m=0$ for $d=1$, and by (2.7) it also holds for $d>1$.

Next we prove (2.2) for $m=1$, the first difference. We first note that the conclusion is trivial for $|t| \leq h^{2}$, since $\left\|D_{\nu}^{ \pm}\right\|=2 / h \leq 2|t|^{-1 / 2}$. Thus we assume hereafter that $|t| \geq h^{2}$. In view of (2.4) and (2.7), it is enough to show that with $d=1$, for $t \in \mathbb{T}_{M}$ and $|t| \geq h^{2}$,

$$
\sum_{j \in \mathbb{Z}}\left|D^{+} g(j h, t)\right| \leq C / \sqrt{|t|} .
$$

We start with the representation

$$
\left(D^{+} g\right)(j h, t)=\frac{i}{h \pi} \int_{-\pi}^{\pi} \sin (\xi / 2) \hat{g}(\xi, t) e^{i(j+1 / 2) \xi} d \xi .
$$

Estimating as in (2.16), and noting the extra factor $|\xi / h|=|s| / \sqrt{|t|}$, we have

$$
\left|\left(D^{+} g\right)(j h, t)\right| \leq C h /|t| .
$$

As in (2.15) we have for $|t| \geq h^{2}$,

$$
\left|\left(\frac{\partial}{\partial \xi}\right)^{2} \sin (\xi / 2) \hat{g}(\xi, t)\right| \leq C \frac{|\xi t|}{h^{2}}\left(1+\frac{\xi^{2}|t|}{h^{2}}\right) e^{-c \xi^{2}|t| / h^{2}} .
$$

In place of (2.17) we find for $j \neq 0$

$$
\left|\left(D^{+} g\right)(j h, t)\right| \leq C /\left(j^{2} h\right) .
$$

Using (2.21) and (2.23), proceeding as in (2.18), we obtain (2.19).

Finally, having established (2.2) for $m=0$ or 1 , we consider higher $m$. Since each $D_{\nu}^{ \pm}$commutes with $\Delta_{h}$, and therefore $e^{\Delta_{h} t}$, we can write symbolically

$$
\left\|D^{2} e^{\Delta_{h} t}\right\|=\left\|\left(D e^{\Delta_{h} t / 2}\right)\left(D e^{\Delta_{h} t / 2}\right)\right\| \leq 2 C_{1}^{2} /|t|
$$

using the case $m=1$. This verifies (2.2) with $m=2$, and $m>2$ can be handled similarly.

TheOrem 2.2. For any $\delta>0$ and all $z$ in the sector

$$
S_{\delta}=\mathbb{C}-\left(\left\{z=z_{1}+i z_{2}: z_{1}<0,\left|z_{2}\right|<\delta\left|z_{1}\right|\right\} \cup\{0\}\right)
$$

we have the estimates, with constants depending on $\delta$ but not on $h$,

$$
\begin{gathered}
\left\|\left(z-\Delta_{h}\right)^{-1}\right\| \leq C_{0}|z|^{-1} ; \\
\left\|D_{h}\left(z-\Delta_{h}\right)^{-1}\right\| \leq C_{1}|z|^{-1 / 2} ; \\
\left\|D_{h}^{2}\left(z-\Delta_{h}\right)^{-1}\right\| \leq C_{2}(1+|\log | z||+|\log h|) .
\end{gathered}
$$

Proof. . We begin with the representation for $z \in \mathbb{C}$ with $\operatorname{Re} z>0$

$$
\left(z-\Delta_{h}\right)^{-1}=\int_{6}^{\infty} e^{-z t} e^{\Delta_{h} t} d t
$$


For $z$ in the sector $S_{1}=\left\{z: z_{1}>0,\left|z_{2}\right| \leq \delta z_{1}\right\}$, we have $\left|e^{-z t}\right|=e^{-z_{1} t} \leq e^{-c|z| t}$ for $t>0$, with $c>0$ depending on $\delta$. Using this and the boundedness of $e^{\Delta_{h} t}$ in (2.29), we obtain (2.26). Similarly, using (2.2) with $m=1$, we have

$$
\left\|D_{h}\left(z-\Delta_{h}\right)^{-1}\right\| \leq C \int_{0}^{\infty} \frac{e^{-c|z| t}}{\sqrt{t}} d t \leq C^{\prime}|z|^{-1 / 2} .
$$

For the second difference, we proceed similarly but split the time interval. For $t \leq h^{2}$ we estimate $D^{\alpha}$ by $h^{-2}$ to obtain

$$
\left\|D_{h}^{2}\left(z-\Delta_{h}\right)^{-1}\right\| \leq C h^{2} h^{-2}+C^{\prime} \int_{h^{2}}^{\infty} \frac{e^{-c|z| t}}{t} d t \leq C^{\prime \prime}\left(1+|\log | z\left|h^{2}\right|\right)
$$

which gives (2.28). We have proved the estimates for $z \in S_{1}$.

Now let $M=2 / \delta$. For any $z=z_{1}+i z_{2}$ with $z_{1}>0$ and $z_{2} \geq 0$, and for $t=t_{1}+i t_{2}$ with $t_{1} \geq 0, t_{2} \leq 0$, we have $\operatorname{Re} t z=t_{1} z_{1}-t_{2} z_{2} \geq t_{1} z_{1}$ and $\left|e^{-z t}\right| \leq e^{-z_{1} t_{1}}$, so that we may deform the path of integration in (2.29) to the ray $R_{2}=\left\{t=t_{1}-i M t_{1}, t_{1} \geq 0\right\}$. This new integral extends the resolvent as an analytic function of $z$ to the sector $S_{2}=\left\{z: z_{2}>0,\left|z_{1}\right| \leq z_{2} / \delta\right\}$, since for such $z$ and for $t \in R_{2}$ we have $\operatorname{Re} t z=$ $t_{1}\left(z_{1}+M z_{2}\right) \geq z_{2} t_{1} / \delta \geq c|z||t|$ for some $c>0$, so that $\left|e^{-z t}\right| \leq e^{-c|z||t|}$. We can now estimate for $z \in S_{2}$ as before, using (2.2) with $t \in R_{2}$. The sector $S_{3}$ conjugate to $S_{2}$ can be handled similarly, and since $S_{\delta}=S_{1} \cup S_{2} \cup S_{3}$, the proof of (2.26)-(2.28) is complete.

3. The discrete Laplacian on a periodic grid. We now consider the problem (1.6) with spatially periodic grid functions. We assume for convenience the period is $2 \pi$ and the interval $[-\pi, \pi]$ is discretized by the set $I_{h}$ of $N$ points. where $N$ is even, $h=2 \pi / N, I=\{j \in \mathbb{Z}:-N / 2<j \leq N / 2\}$, and $I_{h}=\{j h: j \in I\}$. Similarly we discretize $[-\pi, \pi]^{d} \subseteq \mathbb{R}^{d}$ by the grid $I_{h}{ }^{d}$. Let $X_{h}$ be the subspace of $L^{\infty}\left(I_{h}{ }^{d}\right)$ whose elements have mean value zero. We can consider the problem (1.6) on $X_{h} \times[0, \infty)$ as a special case of that on $L^{\infty}\left(\mathbb{R}_{h}^{d}\right) \times[0, \infty)$ and the operator $\Delta_{h}$ on $X_{h}$ as the restriction of $\Delta_{h}$ on $L^{\infty}\left(\mathbb{R}_{h}^{d}\right)$. Similarly $\left(z-\Delta_{h}\right)^{-1}$, when it exists, maps $X_{h}$ to itself and thus is the resolvent of $\Delta_{h}$ on $X_{h}$. Thus the estimates (2.2), (2.26)-(2.28) hold on $X_{h}$ but can be improved. The important difference is that the resolvent set includes $z=0$, that is, $\left(\Delta_{h}\right)^{-1}$ exists on $X_{h}$. We first show exponential decay for the solution operator for $t>0$ on $X_{h}$ and then estimate $\left(\Delta_{h}\right)^{-1}$ and its differences.

Theorem 3.1. There exists $c>0$ so that on the space $X_{h}$,

$$
\left\|D_{h}^{\alpha} e^{\Delta_{h} t}\right\| \leq C_{m} t^{-m / 2} e^{-c t}, \quad t>0, \quad|\alpha|=m, \quad m \geq 0 .
$$

Proof. We will consider only $t \geq 1$, since the conclusion follows from this case and (2.2). We introduce a periodic Green's function with mean value zero,

$$
g_{t}=\Delta_{h} g, \quad g(j h, 0)=\delta_{j 0}-N^{-d} .
$$

The solution of (1.6) for $u^{0} \in X_{h}$ is given as in (2.4) but with $\ell \in I^{d}$. We use the discrete transform

$$
\begin{gathered}
\hat{g}(k, t)=\sum_{j \in I^{d}} g(j h, t) e^{-i k j h}, \quad k \in I^{d}, \\
g(j h, t)=(2 \pi)^{-d} \sum_{k \in I^{d}} \hat{g}(k, t) e^{i k j h} h^{d}, \quad j \in I^{d} .
\end{gathered}
$$


We have $\hat{g}(k, 0)=1$ for $k \neq 0$ and $\hat{g}(0,0)=0$, so that $\hat{g}(k, t)=e^{-\sigma t / h^{2}}$ for $k \neq 0$, $\sigma=\sigma(k h)$, and $\hat{g}(0, t)=0$, where now, in place of $(2.10)$,

$$
\sigma(k h)=4 \sum_{\nu=1}^{d} \sin ^{2}\left(k_{\nu} h / 2\right) .
$$

Thus

$$
g(j h, t)=N^{-d} \sum_{k \neq 0} e^{-\sigma t / h^{2}} e^{i k j h} .
$$

In contrast to the earlier case, $\hat{g}(k, t)$ decays at a uniform rate,

$$
|\hat{g}(k, t)| \leq e^{-c k^{2} t}, \quad k \in I^{d}, k \neq 0, t>0
$$

for some $c>0$, because of (3.5) and (2.13). For $t \geq 1$ and any $j \in I^{d}$,

$$
|g(j h, t)| \leq N^{-d} \sum_{0 \neq k \in I^{d}} e^{-c k^{2} t}=N^{-d} e^{-c t} \sum_{0 \neq k \in I^{d}} e^{-c\left(k^{2}-1\right) t} \leq C N^{-d} e^{-c t} .
$$

Then

$$
\sum_{j \in I^{d}}|g(j h, t)| \leq N^{d} \cdot C N^{-d} e^{-c t}=C e^{-c t} .
$$

The estimate (3.1) with $m=0$ follows from this and the convolution form of the solution operator (2.4), with sum over $I^{d}$.

For the case $m=1$, we suppose $D_{1}^{+}$, a difference in the first coordinate, is applied to $g$. To prove (3.1) in this case it will be enough to verify, in analogy with (3.9), that

$$
\sum_{j \in I^{d}}\left|D_{1}^{+} g(j h, t)\right| \leq \frac{C}{\sqrt{t}} e^{-c t}, \quad t \geq 1
$$

From (3.3) and (3.4),

$$
\begin{gathered}
\left|\left(D_{1}^{+} g\right)^{\wedge}(k)\right| \leq\left|k_{1}\right| e^{-c k^{2} t} \\
\left|D_{1}^{+} g(j h, t)\right| \leq N^{-d} \sum_{k \in I^{d}}\left|k_{1}\right| e^{-c k^{2} t}=N^{-d}\left(\sum_{k_{1} \in I}\left|k_{1}\right| e^{-c k_{1}^{2} t}\right) \ldots\left(\sum_{k_{d} \in I} e^{-c k_{d}^{2} t}\right) .
\end{gathered}
$$

For $t \geq 1$ each factor above except the first is bounded by a constant. For the first we write

$$
\begin{gathered}
\sum_{k_{1} \in I}\left|k_{1}\right| e^{-c k_{1}^{2} t}=2 e^{-c t}+2 \frac{e^{-c t}}{\sqrt{c t}} S_{1} \\
S_{1}=\sum_{k_{1}=2}^{\infty} k_{1} \sqrt{c t} e^{-c\left(k_{1}^{2}-1\right) t} \leq 2 \sum_{k_{1}=2}^{\infty} \sqrt{\left(k_{1}^{2}-1\right) c t} e^{-\left(k_{1}^{2}-1\right) c t} .
\end{gathered}
$$

This sum is decreasing in $t$ for $t \geq 1 / c$, since the function $x e^{-x^{2}}$ is decreasing for $x \geq 1$. Thus the sum is bounded independent of $t \geq 1$. The conclusion (3.10) follows as in (3.9). For higher $m,(3.1)$ again follows from $m=1$. 
Corollary 3.2. For $\Delta_{h}$ on $X_{h}$, we have the estimates

$$
\begin{gathered}
\left\|\left(\Delta_{h}\right)^{-1}\right\| \leq C_{0}, \quad\left\|D_{h}\left(\Delta_{h}\right)^{-1}\right\| \leq C_{1} ; \\
\left\|D_{h}^{2}\left(\Delta_{h}\right)^{-1}\right\| \leq C_{2}(1+|\log h|) .
\end{gathered}
$$

To derive this, we use (3.1) to write

$$
\left(-\Delta_{h}\right)^{-1}=\int_{0}^{\infty} e^{\Delta_{h} t} d t
$$

and estimate directly as in (2.29)-(2.31).

For $z$ near 0 we can write $\left(z-\Delta_{h}\right)^{-1}=\left(-\Delta_{h}\right)^{-1}\left(1-z \Delta_{h}^{-1}\right)^{-1}$. We can then improve the earlier estimates for the space $X_{h}$, replacing $|z|$ in $(2.26),(2.27)$ with $(1+|z|)$, and $|\log z|$ in $(2.28)$ with $\log (|z|+1)$.

4. The Main Regularity Theorem. We suppose a time discretization is chosen for the initial value problem (1.6) with time step $k$ and solution operator at time $n$ of the form $s_{n}\left(k \Delta_{h}\right) u_{0}$, where $s_{n}$ is a function depending on the method. This operator is meaningful provided $s_{n}$ is analytic on the spectrum of $k \Delta_{h}$. For single step methods $s_{n}(z)=s_{1}(z)^{n}$. We derive estimates for the time step operators on $L^{\infty}\left(\mathbb{R}_{h}^{d}\right)$ in which $\Delta_{h}$ is replaced by a general operator with resolvent estimates like (2.26)-(2.28) and with general assumptions for the functions $s_{n}$.

We suppose for each $h>0$ we have a bounded operator $A_{h}$ on $L^{\infty}\left(\mathbb{R}_{h}^{d}\right)$ with spectrum in a sector about the negative real axis, specifically

$$
\left\|A_{h}\right\| \leq C h^{-2}, \quad \operatorname{spec}\left(A_{h}\right) \subseteq \Sigma_{\delta^{\prime}}
$$

for some $0<\delta^{\prime}<\delta$ where

$$
\Sigma_{\delta}=\left\{z=z_{1}+i z_{2}: z_{1} \leq 0,\left|z_{2}\right| \leq \delta\left|z_{1}\right|\right\} .
$$

We assume that on the complementary sector $S_{\delta}$ of $(2.25)$ we have resolvent estimates

$$
\begin{gathered}
\left\|\left(z-A_{h}\right)^{-1}\right\| \leq C_{0}|z|^{-1} ; \quad\left\|D_{h}\left(z-A_{h}\right)^{-1}\right\| \leq C_{1}|z|^{-1 / 2} ; \\
\left\|D_{h}^{2}\left(z-A_{h}\right)^{-1}\right\| \leq C_{2}(1+|\log | z \|+|\log h|)
\end{gathered}
$$

as in (2.26)-(2.28). We assume there is an open set in $\mathbb{C}$, containing $\Sigma_{\delta}$ and a neighborhood of $\infty$, on which each function $s_{n}$ is analytic, $n \geq 1$, and

$$
s_{n}(\infty)=0 .
$$

We assume there is a disk about $0 \in \mathbb{C}$ and some $c_{0}, C_{0}>0$ so that for all $z$ in the disk and each $n$,

$$
\left|s_{n}(z)\right| \leq C_{0}\left(1+c_{0}|z|\right)^{n} .
$$

Finally, we assume there exist $c_{1}, C_{1}>0$ and $p>0$ so that for all $z \in \Sigma_{\delta}$ and all $n$

$$
\left|s_{n}(z)\right| \leq C_{1}\left(1+c_{1}|z|\right)^{-p n} .
$$

THEOREM 4.1. With assumptions (4.1)-(4.7) for $A_{h}$ and $s_{n}$, we have the following estimates for the operators $s_{n}\left(k A_{h}\right)$ and their differences acting on $L^{\infty}\left(\mathbb{R}_{h}^{d}\right)$.

$$
\begin{gathered}
\left\|s_{n}\left(k A_{h}\right)\right\| \leq C_{0} ; \\
\left\|D_{h} s_{n}\left(k A_{h}\right)\right\| \leq C_{1}(n k)^{-1 / 2} ; \\
\left\|D_{h}^{2} s_{n}\left(k A_{h}\right)\right\| \leq C_{2}(n k)^{-1}(1+|\log h|+|\log n k|)
\end{gathered}
$$


with constants independent of $h$ and $k$ for $0<h, k \leq 1$.

Proof. As in [1] and [23] we write $s_{n}\left(k A_{h}\right)$ as a contour integral and estimate norms using (4.3), (4.4). For a simple closed curve $\Gamma$ in $\mathbb{C}$ enclosing the spectrum of $k A_{h}$, positively oriented and depending on $k, h$, we can write

$$
s_{n}\left(k A_{h}\right)=\frac{1}{2 \pi i} \int_{\Gamma} s_{n}(z)\left(z-k A_{h}\right)^{-1} d z
$$

(see e.g. [4], section VII.3). For reference we rewrite (4.3), (4.4) in terms of $k A_{h}$ : for $k \leq 1$ and $z \in S_{\delta}$

$$
\begin{gathered}
\left\|\left(z-k A_{h}\right)^{-1}\right\| \leq C_{0}|z|^{-1} . \\
\left\|D_{h}\left(z-k A_{h}\right)^{-1}\right\| \leq C|k z|^{-1 / 2} . \\
\left\|D_{h}^{2}\left(z-k A_{h}\right)^{-1}\right\| \leq C k^{-1}(1+|\log h|+|\log (|z| / k)|) .
\end{gathered}
$$

Choosing a curve $\Gamma$ to include segments on the rays $z=z_{1}+i z_{2}, z_{1}<0, z_{2}= \pm \delta z_{1}$, and an arc of a circle enclosing $z=0$, we can extend the rays to infinity, using (4.12) and (4.7). In this way we replace $\Gamma$ by a path $\Gamma_{n}$, depending on $n$ but not on $h, k \leq 1$, consisting of the arc

$$
R_{0}=\left\{z=(\varepsilon / n) e^{i \theta},|\theta| \leq \theta_{0}\right\}
$$

and the two rays

$$
R_{ \pm}=\left\{r e^{ \pm i \theta_{0}}, r \geq \varepsilon / n\right\}
$$

Here $\varepsilon$ is chosen small enough so that (4.6) holds for $|z| \leq \varepsilon$, with $\tan \theta_{0}=-\delta$ and $\pi / 2<\theta_{0}<\pi$. We assume for now that $n>2 / p$ and return to the remaining cases later.

We first verify (4.8). For $z \in \Gamma_{n}$ and $k \leq 1$, using (4.12) and (4.6), we estimate the portion of the integral (4.11) on $R_{0}$ by $\left(1+\varepsilon c_{0} / n\right)^{n} \cdot(n / \varepsilon) \cdot 2 \pi(\varepsilon / n) \leq C$. Using (4.12) and (4.7) we estimate the portion on $R_{ \pm}$by

$$
\begin{gathered}
\int_{\varepsilon / n}^{\infty}\left(1+c_{1} r\right)^{-p n} r^{-1} d r \leq \frac{n}{\varepsilon} J, \\
J=\int_{0}^{\infty}\left(1+c_{1} r\right)^{-p n} d r=\frac{1}{c_{1}(p n-1)} \leq \frac{C}{n} .
\end{gathered}
$$

so that this portion is bounded, and (4.8) is established.

For (4.9) we proceed similarly, starting with the integral

$$
D_{h}^{\alpha} s_{n}\left(k A_{h}\right)=\frac{1}{2 \pi i} \int_{\Gamma_{n}} s_{n}(z) D_{h}^{\alpha}\left(z-k A_{h}\right)^{-1} d z
$$

with $|\alpha|=1$. In estimating the portion on $R_{0}$, we now have a factor of $(n /(\varepsilon k))^{1 / 2}$ from (4.13) in place of the earlier factor $(n / \varepsilon)$, resulting in a bound of $C(n k)^{-1 / 2}$. For the portion on $R_{ \pm}$we estimate as in $(4.17)$, $(4,18)$, with $r^{-1}$ in (4.17) replaced by $(r k)^{-1 / 2}$, again from (4.13), and in a similar way we obtain the bound (4.9).

To prove (4.10) we start with the integral (4.19) with $|\alpha|=2$. We estimate the integral on $R_{0}$ using (4.14), with $|z| / k=\varepsilon /(n k)$, as

$$
C(n k)^{-1}(1+|\log h|+|\log n k|) \text {. }
$$


On $R_{ \pm}$we write $|z| / k=n r /(n k)$ and estimate the integral by

$$
k^{-1} \int_{\varepsilon / n}^{\infty}\left(1+c_{1} r\right)^{-p n}(1+|\log h|+|\log n k|+|\log n r|) d r .
$$

The factor $(1+|\log h|+|\log n k|)$ multiplies a term bounded by $k^{-1} J \leq C(n k)^{-1}$, according to (4.18), and this part of (4.21) is bounded as in (4.20). In the last term we split the integral, presuming $\varepsilon<1$,

$$
k^{-1} \int_{\varepsilon / n}^{\infty}\left(1+c_{1} r\right)^{-p n}|\log n r| d r=k^{-1} \int_{\varepsilon / n}^{1 / n}+k^{-1} \int_{1 / n}^{\infty} .
$$

For $\varepsilon \leq n r \leq 1,|\log n r| \leq|\log \varepsilon|$, a constant, so that the first term in (4.22) is bounded by $C(n k)^{-1}$. For the second term, we use the convexity of the function $x \mapsto x^{n p / 2}$ with $n p / 2>1$ to say that $\left(1+c_{1} r\right)^{n p / 2} \geq 1+c_{1} p n r / 2$. For $n r \geq 1$, $|\log n r| /\left(1+c_{1} p n r / 2\right)$ is bounded, and we can now estimate

$$
k^{-1} \int_{1 / n}^{\infty}\left(1+c_{1} r\right)^{-p n}|\log n r| d r \leq C k^{-1} \int_{1 / n}^{\infty}\left(1+c_{1} r\right)^{-p n / 2} d r \leq C^{\prime}(n k)^{-1}
$$

the last as in (4.18). This completes the proof of (4.10).

It remains to prove (4.8)-(4.10) for $n \leq n_{0}$, where $n_{0}>2 / p$ is fixed. Since the decay in (4.7) for large $z$ is slow for such $n$, we subtract a term from $s_{n}(z)$ to improve the convergence in $(4.11),(4.19)$. Since $s_{n}(\infty)=0$, we have $s_{n}(z)=a_{n} z^{-1}+O\left(|z|^{-2}\right)$ for some $a_{n}$. We set $\tilde{s}_{n}(z)=s_{n}(z)+a_{n}(1-z)^{-1}$, so that $\tilde{s}_{n}(z)=O\left(|z|^{-2}\right)$ as $z \rightarrow \infty$, and thus for some $c, C$

$$
\left|\tilde{s}_{n}(z)\right| \leq C(1+c|z|)^{-2}, \quad z \in \Sigma_{\delta} .
$$

Since $s_{n}(z)=\tilde{s}_{n}(z)-a_{n}(1-z)^{-1}$,

$$
s_{n}\left(k A_{h}\right)=\tilde{s}_{n}\left(k A_{h}\right)-a_{n}\left(1-k A_{h}\right)^{-1} .
$$

From (4.12)-(4.14), with $z=1$, we see that $\left(1-k A_{h}\right)^{-1}$ satisfies (4.8)-(4.10); here we note that $k^{-1} \leq n_{0}(n k)^{-1}=C(n k)^{-1}$. We can estimate $\tilde{s}_{n}\left(k A_{h}\right)$ as before for $s_{n}\left(k A_{h}\right)$, using the integral representation, with (4.24) in place of (4.7). The modification is straightforward since $n$ is bounded.

5. Proofs of Theorems 1.1 and 1.2. We prove Theorems 1.1 and 1.2 from Theorem 4.1. For Theorem 1.1 we verify that the hypotheses on $s$ imply those for $s_{n}=s^{n}$ in Theorem 4.1. The application to TGA and SDIRK2 is explained.

Proof of Theorem 1.1. First, since the time discretization is consistent, we must have

$$
s(z)=1+z+O\left(z^{2}\right) \quad \text { as } z \rightarrow 0 .
$$

This implies that $|s(z)| \leq 1+c_{0}|z|$ for $z$ in some disk about 0 and for some $c_{0}$, so that (4.6) holds with $C_{0}=1$. We will show that (4.7) holds with $p=1$ and $C_{1}=1$; that is, we show for some $c_{1}>0$,

$$
|s(z)| \leq\left(1+c_{1}|z|\right)^{-1}, \quad z \in \Sigma_{\delta} .
$$


Here $\delta>0$ is chosen so that $s$ is analytic on a neighborhood of $\Sigma_{\delta}$ and $|s(z)| \leq 1$ on a larger sector. We consider three parts of $\Sigma_{\delta}$, starting with $z$ small. For $z=z_{1}+i z_{2} \in$ $\Sigma_{\delta}$, we have $|1+z|^{2}=\left(1+2 z_{1}\right)+|z|^{2}, z_{1} \leq 0$, and $|z| \leq\left(1+\delta^{2}\right)^{1 / 2}\left(-z_{1}\right)$. From these facts and (5.1) we can see that $|s(z)| \leq\left(1-c_{1}|z|\right) \leq\left(1+c_{1}|z|\right)^{-1}$ for $|z| \leq \varepsilon$, provided $c_{1} \leq\left(1+\delta^{2}\right)^{-1 / 2} / 2$ and $\varepsilon$ is small enough. Next we consider $z$ large. Since $s$ is analytic at $\infty$ and $s(\infty)=0$, we have $s(z)=a / z+O\left(|z|^{-2}\right)$. Then there exist $r_{0}, M>0$ so that $|s(z)| \leq M /|z|$ for $|z| \geq r_{0}$. Let $r_{1}=\max \left(r_{0}, 2 M\right)$ and $c_{1}=1 /(2 M)$. Then for $|z| \geq r_{1},\left(1+c_{1}|z|\right) \leq|z| / M$, so that $\left(1+c_{1}|z|\right)^{-1} \geq M /|z| \geq|s(z)|$. We have now verified (5.2) for $z$ small or large, and we are left with the compact set $\Sigma_{\text {mid }}=\left\{z \in \Sigma_{\delta}: \varepsilon \leq|z| \leq r_{1}\right\}$. Since this set has a neighborhood where $s$ is analytic and $|s(z)| \leq 1$, it follows from the Maximum Modulus Principle that $|s(z)|<1$ on $\Sigma_{\text {mid }}$. Then there exists $\kappa<1$ so that $|s(z)| \leq \kappa$ for $z \in \Sigma_{\text {mid }}$, and we can choose $c_{1}$ sufficiently small so that $\kappa<\left(1+c_{1} r_{1}\right)^{-1}$. Thus (5.2) holds on $\Sigma_{\text {mid }}$ as well, and the inequality is verified for all of $\Sigma_{\delta}$.

Proof of Theorem 1.2. Let $M=\sup _{t \leq T}\|f(\cdot, t)\|$, and denote the right side of (1.11) by $k \tilde{f}^{n}$. The solution of (1.11) is

$$
u^{n}=\sum_{\ell=0}^{n-1} s\left(k \Delta_{h}\right)^{n-1-\ell} \tilde{f}^{\ell} k .
$$

From (4.8) and (4.12) with $z=1$, we have $\left\|u^{n}\right\| \leq \sum C M k=C M n k \leq C^{\prime} M$. For first differences, we note that (4.13) implies $\left\|D_{h}\left(1-\eta_{i} k \Delta_{h}\right)^{-1} f\right\| \leq C M k^{-1 / 2}$. Using this for $\ell=n-1$ and (4.9) for $\ell<n-1$ we get, with $\ell \mapsto n-1-\ell$,

$$
\left\|D_{h} u^{n}\right\| \leq C M k^{1 / 2}+C M \sum_{\ell=1}^{n-1}(\ell k)^{-1 / 2} k \leq C^{\prime} M(n k)^{1 / 2} \leq C^{\prime} M T^{1 / 2} .
$$

For $D_{h}^{2}$, (4.14) gives $\left\|D_{h}^{2}\left(1-\eta_{i} k \Delta_{h}\right)^{-1} f\right\| \leq C M k^{-1}(1+|\log h|)$. We use this for $\ell=n-1$ and otherwise we use (4.10) to obtain

$$
\begin{aligned}
\left\|D_{h}^{2} u^{n}\right\| & \leq C M(1+|\log h|)\left(1+\sum_{\ell=1}^{n-1}(\ell k)^{-1} k\right) \\
\leq & C M(1+|\log h|)(1+|\log T|+|\log k|) \leq C^{\prime} M\left(1+|\log h|^{2}\right)
\end{aligned}
$$

TGA and SDIRK2. We verify that Theorems 1.1 and 1.2 apply to these two methods among others. Each is $L$-stable. We can see from (A.3) and (A.6)-(A.8) that each operator $q_{i}\left(k \Delta_{h}\right)$ in (1.11) has the form

$$
q_{i}\left(k \Delta_{h}\right)=p\left(k \Delta_{h}\right)\left(1-a_{1} k \Delta_{h}\right)^{-1} \ldots\left(1-a_{\ell} k \Delta_{h}\right)^{-1}
$$

where $p$ is a polynomial, $a_{j}>0$ for $1 \leq j \leq \ell$, and $\ell \geq \operatorname{deg} p$. Now (2.26) implies that the operator $\left(1-a_{j} k \Delta_{h}\right)^{-1}$ on $L^{\infty}$ has norm bounded by $C_{0}$. It follows that any operator $\left(b_{0}+b_{1} k \Delta_{h}\right)\left(1-a_{j} k \Delta_{h}\right)^{-1}$ is bounded on $L^{\infty}$ uniformly in $h$ and $k$, and thus $q_{i}\left(k \Delta_{h}\right)$, being a product of bounded factors, is also uniformly bounded on $L^{\infty}$. Thus the hypotheses of the two theorems are satisfied. The same remark applies to any other $L$-stable single-step method with equation (1.11) provided $q_{i}\left(k \Delta_{h}\right)$ has the form just described. 
6. The backward difference formula. We verify that Theorem 4.1 applies to BDF2, as given in (A.11). As a consequence, Theorem 1.2 holds for BDF2 as well. We suppose here that the two-step BDF2 method is started with one step of the backward Euler formula.

If we replace $\Delta_{h}$ with $\lambda \in \mathbb{C}$ and $u^{n}$ with $y_{n}$ in (A.11), we have the iteration

$$
\frac{3}{2} y_{n+1}-2 y_{n}+\frac{1}{2} y_{n-1}=k \lambda y_{n+1}
$$

or, with $z=k \lambda$,

$$
\left(\begin{array}{c}
y_{n+1} \\
y_{n}
\end{array}\right)=M(z)\left(\begin{array}{c}
y_{n} \\
y_{n-1}
\end{array}\right), \quad M(z)=\left(\begin{array}{cc}
2\left(\frac{3}{2}-z\right)^{-1} & -\frac{1}{2}\left(\frac{3}{2}-z\right)^{-1} \\
1 & 0
\end{array}\right) .
$$

With $y_{0}, y_{1}$, specified, $y_{n}(z)$ is analytic for Re $z \leq 0$. The general solution of (6.1) is

$$
y_{n}=c_{+} w_{+}^{n}+c_{-} w_{-}^{n}, \quad w_{ \pm}=(2 \pm \sqrt{1+2 z}) /(3-2 z) .
$$

It is well-known that BDF2 is A-stable ([8], Sec V.1; [9] Sec. 4.4), i.e.,

$$
\left|w_{ \pm}(z)\right| \leq 1, \quad \operatorname{Re} z<0 .
$$

(The double root at $z=-1 / 2$ will not concern us directly.)

Since we start with one backward Euler step, we take $y_{0}=1$ and $y_{1}=(1-z)^{-1}$. Then $y_{n}$ corresponds to $s_{n}(z)$ in the Theorem, and $s_{n}(z)=c_{+} w_{+}^{n}+c_{-} w_{-}^{n}$ with

$$
c_{+}=\left(-w_{-}+y_{1}\right) /\left(w_{+}-w_{-}\right), \quad c_{-}=\left(w_{+}-y_{1}\right) /\left(w_{+}-w_{-}\right), \quad y_{1}=(1-z)^{-1} .
$$

We now check the hypotheses of the Theorem. From (6.2) we can regard $s_{n}(z)$ as the first component of $M(z)^{n-1}\left((1-z)^{-1} 1\right)^{T}$. It is analytic on any sector $\Sigma_{\delta}$ and at infinity. Since

$$
M(z)^{2}=\left(\frac{3}{2}-z\right)^{-1}\left(\begin{array}{cc}
4-\left(\frac{3}{2}-z\right)^{-1} / 2 & -1 \\
2 & -\frac{1}{2}
\end{array}\right)
$$

it is apparent that $s_{n}(\infty)=0$. For $z$ near 0 , we choose the positive square root, so that $w_{+}(z)=1+z+z^{2} / 2+O\left(z^{3}\right)$, while $w_{-}(0)=1 / 3$. From (6.5), $c_{+}=1+O\left(z^{2}\right)$, $c_{-}=O\left(z^{2}\right)$. It is now evident that (4.6) holds for some $C_{0}, c_{0}$ and $z$ in a disk about 0 . It is also evident, as in the discussion of the single step method, that (4.7) holds for $z$ near 0 with $p=1$ and therefore also $p \leq 1$. For large $z, w_{ \pm} \approx \pm 1 / \sqrt{2 z}$ and $c_{ \pm} \rightarrow 1 / 2$. From this and (6.3),

$$
\left|s_{n}(z)\right| \leq C|z|^{-n / 2}, \quad z \text { large } .
$$

Now on the rays $z=z_{1} \pm \delta z_{1}, z_{1}<0$, we have $\left|w_{ \pm}\right| \leq 1$ by the stability condition (6.4), and $c_{ \pm}$are bounded. Thus $s_{n}(z)$ is bounded independent of $n$ on these rays, and by its analyticity it is also bounded inside the sector. We can now verify (4.7) with $p=1 / 2$, using the boundedness in the sector, (6.7) for large $z$, and the case of small $z$ already checked, in a manner similar to the proof in Theorem 1.1 for a single-step method.

The proof of Theorem 1.2 is easily modified for BDF2, writing the nonhomogeneous equation in a form like (6.2), and noting $f^{n}$ is multiplied by $\left(3 / 2-k \Delta_{h}\right)^{-1}$ as in (1.11). 
7. The Crank-Nicolson method. For the Crank-Nicolson, or trapezoidal, discretization of (1.10), stated in (A.1), (A.2), Theorem 4.1 does not apply since $s(\infty) \neq 0$. It can be seen easily from examples that the analogues of (1.8), (1.9) and (1.13) do not hold for CN with $k=c h$, since $s\left(k \Delta_{h}\right)=-1+O(h)$ in the highest modes. We prove a weaker version of (1.8) and the smoothing property (1.12) for the nonhomogeneous problem. The boundedness of $s\left(k \Delta_{h}\right)^{n}$ follows from [19], but we give a brief proof here.

THEOREM 7.1. With $A_{h}$ as in Theorem 4.1 and s given by (A.2) we have

$$
\begin{gathered}
\left\|s\left(k A_{h}\right)^{n}\right\| \leq C_{0}, \\
\left\|D_{h} s\left(k A_{h}\right)^{n}\left(1-k A_{h} / 2\right)^{-1}\right\| \leq C_{1}(n k)^{-1 / 2} .
\end{gathered}
$$

Proof. We first estimate $|s(z)|$ for $z \in \Sigma_{\delta}$. For such $z=z_{1}+i z_{2}$ we have $z_{1} \leq 0$, $|z| \leq C\left|z_{1}\right|,|1+z / 2|^{2}=|1-z / 2|^{2}+2 z_{1}$ or $|s(z)|^{2}=1+2 z_{1} /|1-(z / 2)|^{2}$, and

$$
|s(z)| \leq 1+\frac{z_{1}}{|1-z / 2|^{2}} \leq 1-\frac{c|z|}{1+|z|^{2}}
$$

for some $c>0$. We will use the two consequences

$$
\begin{array}{cl}
|s(z)| \leq 1-c_{0}|z|, & |z| \leq 1, \quad z \in \Sigma_{\delta} \\
|s(z)| \leq 1-c_{1} /|z|, & |z| \geq 1, \quad z \in \Sigma_{\delta} .
\end{array}
$$

On the other hand, for any large $z$ we have

$$
|s(z)| \leq 1+8 /|z|, \quad|z| \geq 4 .
$$

We now prove (7.2) in a way similar to [1], Lemma 1.1, p. 189. In view of the above, we may write the operator as a Cauchy integral as in (4.11), (4.19),

$$
D_{h} s\left(k A_{h}\right)^{n}\left(1-k A_{h} / 2\right)^{-1}=\frac{1}{2 \pi i} \int_{\Gamma_{n}} s(z)^{n}(1-z / 2)^{-1} D_{h}\left(z-k A_{h}\right)^{-1} d z
$$

using the same $n$-dependent contour as in (4.15), (4.16). On the $\operatorname{arc} R_{0}$ near $z=0$ we have the same estimate as before, since the factor $(1-z / 2)^{-1}$ is bounded away from zero for $z$ small. On the ray $R_{ \pm}$we use the inequality $|1-z / 2| \geq c(1+|z|)$, the resolvent estimate (4.13), and (7.4),(7.5) above. We obtain the upper bound for the integral on $R_{ \pm}$

$$
\begin{gathered}
C \int_{\varepsilon / n}^{\infty} \frac{|s(z)|^{n}}{(1+|z|) \sqrt{k|z|}} d|z| \leq C^{\prime} k^{-1 / 2}\left(I_{1}+I_{2}\right), \\
I_{1}=\int_{1}^{\infty}(1-c / r)^{n} r^{-3 / 2} d r, \quad I_{2}=\int_{0}^{1}(1-c r)^{n} r^{-1 / 2} d r .
\end{gathered}
$$

The change of variables $s=1 / r$ in $I_{1}$ results in the same integral as $I_{2}$. For either we estimate, presuming $c<1$,

$$
\begin{aligned}
& \int_{0}^{1}(1-c r)^{n} r^{-1 / 2} d r \leq \int_{0}^{1 / n} r^{-1 / 2} d r+n^{-1 / 2} \int_{1 / n}^{1}(1-c r)^{n} d r \\
& \leq 2 n^{-1 / 2}+C n^{-1 / 2} n^{-1} \leq C^{\prime} n^{-1 / 2}
\end{aligned}
$$


so that (7.8) is bounded by $C_{1}(n k)^{-1 / 2}$, completing (7.2).

To prove (7.1) we first note that, for each $n, s(z)^{n}-(-1)^{n}=O\left(|z|^{-1}\right)$ as $z \rightarrow \infty$. With this fact and (4.12), we can use an integral representation with the same contour (4.15), (4.16)

$$
s\left(k A_{h}\right)^{n}-(-1)^{n}=\frac{1}{2 \pi i} \int_{\Gamma}\left(s(z)^{n}-(-1)^{n}\right)\left(z-k A_{h}\right)^{-1} d z .
$$

The integrand is $O\left(|z|^{-2}\right)$ for large $z$. To estimate $s\left(k A_{h}\right)^{n}$ we change the contour to a bounded one as in [23], Thm. 9.1. With $\rho>0$ large, we use the closed curve $\Gamma_{\rho}$ consisting of the arc $R_{0}$, the segments on the rays $R_{ \pm}$with $\varepsilon / n \leq|z| \leq \rho$, and the arc $R_{\rho}=\left\{z=\rho e^{i \theta},|\theta| \leq \theta_{0}\right\}$, with $\theta_{0}$ as in (4.15). Since the integrand is $O\left(|z|^{-2}\right)$ for large $z$, in principle we commit an error $O(1 / \rho)$ in using this contour, but in fact the integral is independent of $\rho>2$, by the analyticity of $s$ and $\left(z-k A_{h}\right)^{-1}$. Thus the new integral formula is exact. Moreover, the term $(-1)^{n}$ contributes zero to the integral. We now have

$$
s\left(k A_{h}\right)^{n}=(-1)^{n}+\frac{1}{2 \pi i} \int_{\Gamma_{\rho}} s(z)^{n}\left(z-k A_{h}\right)^{-1} d z .
$$

We choose $\rho_{1}>2$ and $\rho_{n}=n \rho_{1}$. We can now show that the norm is bounded. Using (7.6), (4.12) we bound the integral on $R_{\rho}$ by $C\left(1+8 /\left(n \rho_{1}\right)\right)^{n} \leq C^{\prime}$, and similarly for $R_{0}$, as before. We bound the integrals on the rays, using (4.12), (7.4), (7.5), by a constant times

$$
\int_{1}^{\rho_{n}}\left(1-c_{1} / r\right)^{n} r^{-1} d r+\int_{\varepsilon / n}^{1}(1-c r)^{n} r^{-1} d r
$$

The second integral is bounded by

$$
(n / \varepsilon) \int_{0}^{1}(1-c r)^{n} d r \leq C
$$

while the first converts under the change of variable $s=1 / r$ to an integral with the same integrand, from $1 / \rho_{n}=1 /\left(\rho_{1} n\right)$ to 1 , and is estimated in the same way.

For the nonhomogeneous problem (1.10), the analogue of (1.11) for $\mathrm{CN}$ is

$$
u^{n+1}=s\left(k \Delta_{h}\right) u^{n}+k\left(1-\frac{k}{2} \Delta_{h}\right)^{-1} f^{n+1 / 2} .
$$

Because of the resolvent factor on the right, we can estimate $\left\|u^{n}\right\|,\left\|D_{h} u^{n}\right\|$ using (7.1), (7.2), as in the proof of Theorem 1.2:

COROLlary 7.2. If (1.10) is approximated by (7.15) with $k=c h, 0 \leq n k \leq T$, then $\left\|u^{n}\right\|$ and $\left\|D_{h} u^{n}\right\|$ are bounded as in (1.12).

8. Convection-diffusion with an interface. We consider the approximation of spatially periodic solutions of a convection-diffusion equation

$$
u_{t}+a \cdot \nabla u=\Delta u
$$

where $a=a(x, t) \in \mathbb{R}^{d}$, with discontinuities at an interface. In a rectangular domain $\Omega=(0, L)^{d} \subseteq \mathbb{R}^{d}$, suppose that, for $0 \leq t \leq T, B(t)$ is a subdomain with boundary $\Gamma(t) \subseteq \Omega$, and suppose $\cup_{0 \leq t \leq T}(\Gamma(t) \times\{t\})$ is the image of $S^{d-1} \times[0, T]$ under a $C^{1}$, 
injective, nondegenerate mapping, preserving $t$, where $\frac{S^{d-1}}{B(t)} \times\left\{x \in \mathbb{R}^{d}:|x|=1\right\}$. We will assume that $a$ and $u$ are smooth on the set $\cup_{t} \overline{B(t)} \times\{t\}$ and also smooth on $\cup_{t}(\bar{\Omega}-B(t)) \times\{t\}$ with periodic boundary conditions; jump discontinuities are allowed at $\Gamma(t)$. We suppose that the jumps

$$
u_{+}-u_{-}=g_{0}, \quad \frac{\partial u_{+}}{\partial n}-\frac{\partial u_{-}}{\partial n}=g_{1} \quad \text { on } \Gamma(t)
$$

are specified or otherwise known, where $n$ is the normal vector to $\Gamma(t)$.

We suppose this problem is discretized on a square grid with size $h$, with $L / h$ an integer, and with time step $k=c h$, using any of the schemes discussed here. We suppose the truncation error is $O\left(h^{2}\right)$ away from the interface and is corrected to $O(h)$ near the interface. We show that, with reasonable assumptions, the error in the solution $\varepsilon^{n}$ at time $n k$ is uniformly $O\left(h^{2}\right)$ :

$$
\left\|\varepsilon^{n}\right\| \leq C h^{2} .
$$

Moreover, for TGA, SDIRK2, or BDF2, first spatial differences are almost $O\left(h^{2}\right)$ :

$$
\left\|D_{h} \varepsilon^{n}\right\| \leq C h^{2}\left(1+|\log h|^{2}\right) .
$$

From these differences, accurate values can be obtained for first derivatives, using corrections at the interface.

To be specific, we assume at first that (8.1) is discretized with CN, using an explicit $O\left(h^{2}\right)$ version of $a \cdot \nabla u$ at time $n+1 / 2$,

$$
\frac{u^{n+1}-u^{n}}{k}+\frac{3}{2} a^{n} \cdot \nabla_{h} u^{n}-\frac{1}{2} a^{n-1} \cdot \nabla_{h} u^{n-1}=\frac{1}{2} \Delta_{h} u^{n+1}+\frac{1}{2} \Delta_{h} u^{n}
$$

where $k=c h$ and $\nabla_{h}$ is the centered difference. (A different scheme is needed for $n=0$; see below.) The exact solution has truncation error in the form $\chi^{n}+\tau^{n}$, where $\chi^{n}$ will represent large errors near the interface. We will call $(j h, n k)$ a regular point if the interface does not intersect the stencil of $\Delta_{h}$ centered at $j h$ at time $n k$ or $(n+1) k$, and also does not cross the line segment at $j h$ from $n k$ to $(n+1) k$. For such $j h$ the difference formulas in (8.5) have the usual accuracy; in this case we set $\chi^{n}(j h)=0$, and $\tau^{n}(j h)=O\left(h^{2}\right)$. At an irregular point the largest contribution $\chi^{n}(j h)$ can be found using the jump conditions $(8.2)$, as in $[12,13,17]$, so that the remaining truncation error $\tau^{n}(j h)$ is $O(h)$. Once $\chi^{n}$ is known, the discrete solution can be corrected by adding $\chi^{n}$ to the right side of (8.5). In this way, a discrete solution is obtained so that the error $\varepsilon^{n}$ satisfies equation (8.5) with $\tau^{n}$ on the right, that is,

$$
\left(1-\frac{k}{2} \Delta_{h}\right) \varepsilon^{n+1}=\left(1+\frac{k}{2} \Delta_{h}\right) \varepsilon^{n}-\frac{3}{2} k a^{n} \cdot \nabla_{h} \varepsilon^{n}+\frac{1}{2} k a^{n-1} \cdot \nabla_{h} \varepsilon^{n-1}+k \tau^{n}
$$

or

$$
\varepsilon^{n+1}=s\left(k \Delta_{h}\right) \varepsilon^{n}+k q\left(k \Delta_{h}\right) F^{n}+k q\left(k \Delta_{h}\right) \tau^{n}
$$

where

$$
s(z)=(1+z / 2)(1-z / 2)^{-1}, \quad q(z)=(1-z / 2)^{-1}
$$

and $F^{n}$ represents the convection terms in (8.6). We will need the following lemma, proved at the end of the section. It shows that $\tau^{n}$ is the discrete divergence of an $O\left(h^{2}\right)$ grid function plus remainder: 
LEMma 8.1. If the periodic grid function $\tau^{n}$ is uniformly $O\left(h^{2}\right)$ at regular points and $O(h)$ and irregular points, then there are periodic grid functions $\Phi_{\nu}^{n}, 0 \leq \nu \leq d$, so that

$$
\tau^{n}=\sum_{\nu=1}^{d} D_{\nu}^{-} \Phi_{\nu}^{n}+\Phi_{0}^{n} \quad \text { and } \quad\left\|\Phi_{\nu}^{n}\right\| \leq C h^{2}
$$

We assume the scheme is started at $n=1$ in such a way that $\varepsilon^{0}=0$ and $\left\|\varepsilon^{1}\right\| \leq C h^{2}$. We now have, as in (5.3), for $n \geq 2$,

$$
\varepsilon^{n}=s\left(k \Delta_{h}\right)^{n-1} \varepsilon^{1}+k \sum_{\ell=1}^{n-1} s\left(k \Delta_{h}\right)^{n-1-\ell} q\left(k \Delta_{h}\right)\left(F^{\ell}+\sum_{\nu=1}^{d} D_{h, \nu} \Phi_{\nu}^{\ell}+\Phi_{0}^{\ell}\right)
$$

We estimate $\left\|\varepsilon^{n}\right\|$. From (7.1), $\left\|s\left(k \Delta_{h}\right)^{n-1} \varepsilon^{1}\right\| \leq C_{0}\left\|\varepsilon^{1}\right\| \leq C^{\prime} h^{2}$. We proceed with the other terms as in the proof of Thm. 1.2, beginning with the first convection term. We write $a^{\ell} \cdot \nabla_{h} \varepsilon^{\ell}=\nabla_{h} \cdot\left(a^{\ell} \varepsilon^{\ell}\right)+b^{\ell}$, where $b^{\ell}$ depends boundedly on $\varepsilon^{\ell}$. We commute operators in the main term:

$$
s\left(k \Delta_{h}\right)^{n-1-\ell} q\left(k \Delta_{h}\right) \nabla_{h} \cdot\left(a^{\ell} \varepsilon^{\ell}\right)=\nabla_{h} \cdot s\left(k \Delta_{h}\right)^{n-1-\ell} q\left(k \Delta_{h}\right)\left(a^{\ell} \varepsilon^{\ell}\right) .
$$

For $\ell \leq n-2$ we use (7.2) to bound this by $C((n-1-\ell) k)^{-1 / 2}\left\|\varepsilon^{\ell}\right\|$. For $\ell=n-1$ we use (4.13) to get the bound $C k^{-1 / 2}\left\|\varepsilon^{n-1}\right\|$. Each term $s\left(k \Delta_{h}\right)^{n-1-\ell} q\left(k \Delta_{h}\right) b^{\ell}$ is bounded by $C\left\|\varepsilon^{\ell}\right\|$. For the second convection term we get similar estimates with $\varepsilon^{\ell}$ replaced by $\varepsilon^{\ell-1}$ and $\varepsilon^{n-1}$ by $\varepsilon^{n-2}$. For the terms in (8.10) with $D_{h, \nu} \Phi_{\nu}^{\ell}$ we again commute $D_{h, \nu}$ forward and find, using (7.2) and (4.13), that $s\left(k \Delta_{h}\right)^{n-1-\ell} q\left(k \Delta_{h}\right) D_{h, \nu} \Phi_{\nu}^{\ell}$ is bounded by $C((n-1-\ell) k)^{-1 / 2}\left\|\Phi_{\nu}^{\ell}\right\| \leq C^{\prime} h^{2}((n-1-\ell) k)^{-1 / 2}$ for $\ell \leq n-2$ and $C k^{-1 / 2}\left\|\Phi_{\nu}^{n-1}\right\| \leq C^{\prime} h^{2} k^{-1 / 2}$ for $\ell=n-1$. The contribution to (8.10) from these terms is thus bounded by

$$
C h^{2} k\left(k^{-1 / 2}+\sum_{\ell=1}^{n-2}((n-1-\ell) k)^{-1 / 2}\right) \leq C^{\prime} h^{2}
$$

and the contribution from $\Phi_{0}^{\ell}$ is similar but simpler. Combining the estimates we obtain for $n \geq 2$ (with sum omitted for $n=2,3$ )

$$
\left\|\varepsilon^{n}\right\| \leq C_{1} \sum_{\ell=1}^{n-3}((n-2-\ell) k)^{-1 / 2}\left\|\varepsilon^{\ell}\right\| k+C_{1} k^{-1 / 2}\left(\left\|\varepsilon^{n-1}\right\|+\left\|\varepsilon^{n-2}\right\|\right) k+C_{2} h^{2}
$$

To simplify this, we choose $\alpha, 1<\alpha<2$, so that $t^{-1 / 2} \in L^{\alpha}(0, T)$. With $1 / \beta=1-1 / \alpha$ we apply Hölder's inequality,

$$
\left\|\varepsilon^{n}\right\|^{\beta} \leq C_{1}^{\prime}\left(\sum_{\ell=1}^{n-3}((n-2-\ell) k)^{-\alpha / 2} k+2 k^{-\alpha / 2} k\right)^{\beta / \alpha} \sum_{\ell=1}^{n-1}\left\|\varepsilon^{\ell}\right\|^{\beta} k+C_{2}^{\prime} h^{2 \beta}
$$

The first sum is bounded independent of $n$, and we can now apply a discrete Gronwall inequality (e.g. [23], Lemma 10.5, p. 175) to conclude $\left\|\varepsilon^{n}\right\|^{\beta} \leq C^{\prime} h^{2 \beta}$, thus proving (8.3) for the CN scheme (8.5).

Now suppose we discretize (8.1) as in (8.5) using TGA rather than CN, treating the convection term as before. We obtain an error equation as in (8.7) with different 
$s$ and $q$. Arguing just as above, but using (1.7), (1.8) rather than (7.1), (7.2), we can again verify (8.3). Moreover, we can prove (8.4) in a related way provided we assume the scheme is started carefully enough so that (8.3) and (8.4) hold for $\varepsilon^{1}$. To verify (8.4) we apply $D_{h, \mu}$ to (8.10). We bound the convection term by $\nabla_{h} \varepsilon$ rather than $\varepsilon$, obtaining an estimate for this part similar to the earlier case. For the truncation term we use (1.9) and (4.14) to estimate

$$
D_{h, \mu} s\left(k \Delta_{h}\right)^{n-1-\ell} q\left(k \Delta_{h}\right) D_{h, \nu} \Phi_{\nu}^{\ell}=D_{h, \mu} D_{h, \nu} s\left(k \Delta_{h}\right)^{n-1-\ell} q\left(k \Delta_{h}\right) \Phi_{\nu}^{\ell}
$$

by $C((n-1-\ell) k)^{-1}(1+|\log h|)\left\|\Phi_{\nu}^{\ell}\right\| \leq C^{\prime} h^{2}((n-1-\ell) k)^{-1}(1+|\log h|)$ for $\ell \leq n-2$ and $C k^{-1}(1+|\log h|)\left\|\Phi_{\nu}^{n-1}\right\| \leq C^{\prime} h^{2} k^{-1}(1+|\log h|)$ for $\ell=n-1$. The resulting sum is bounded by $C h^{2}\left(1+|\log h|^{2}\right)$ as in (5.5). Note that (1.9) was not available for CN. Summing over $\mu$, we get

$$
\begin{aligned}
\left\|D_{h} \varepsilon^{n}\right\| \leq C_{1} & \sum_{\ell=1}^{n-3}((n-2-\ell) k)^{-1 / 2}\left\|D_{h} \varepsilon^{\ell}\right\| k \\
& +C_{1} k^{-1 / 2}\left(\left\|D_{h} \varepsilon^{n-1}\right\|+\left\|D_{h} \varepsilon^{n-2}\right\|\right) k+C_{2} h^{2}\left(1+|\log h|^{2}\right)
\end{aligned}
$$

which as before leads to (8.4). Similar estimates could be made for SDIRK2 or BDF2.

We have presumed here that the time-dependent position of the interface was given. In a more realistic problem its position would depend on $u$, and the errors in $u$ and the interface position would be coupled. The estimates here apply only to the error in $u$ due to the treatment of the interface. The control of errors in $\nabla u$ as well as $u$, given by (8.4), can be important in computing the interface motion.

Proof of Lemma 8.1. The proof is similar to that of Lemmas 2.2 and 2.6 in [3] for the time-independent case. Since $k=O(h)$, an irregular point of any kind at time $n k$ must be within $O(h)$ of $\Gamma(n k)$; this fact depends on the boundedness the $t$-derivative of the map defining $\Gamma(t)$. We suppose first that $\tau$ is supported in a coordinate patch; specifically we assume that $\tau(j h, n k)$ is nonzero only for irregular points $(j h, n k)$ in a set where $\Gamma(t)$ can be given by $x_{d}=Y(\tilde{x}, t)$; here $\tilde{x}=\left(x_{1}, \ldots, x_{d-1}\right)$ and $Y$ is $C^{1}$. We claim that with $n$ fixed, for each $\tilde{j} h=\left(j_{1} h, \ldots, j_{d-1} h\right)$, any irregular point $\left(\tilde{j} h, j_{d} h, n k\right)$ in this set, with some $j_{d}$, must have $\left|j_{d} h-Y(\tilde{j} h, n k)\right| \leq C_{0} h$ for some constant $C_{0}$. Thus for each $n$ and $\tilde{j}$, the number of irregular points $\left(\tilde{j} h, j_{d} h, n k\right)$ must be bounded. To prove the claim, suppose we have such an irregular point. Then there is a point on $\Gamma(n k)$ of the form $\left(\tilde{x}^{*}, Y\left(\tilde{x}^{*}\right)\right)$ within $O(h)$. (We omit the dependence on $n k$ for simplicity.) Then $\tilde{x}^{*}-\tilde{j} h=O(h)$; therefore $Y\left(\tilde{x}^{*}\right)-Y(\tilde{j} h)=O(h)$ also, and thus $j_{d} h$ is within $O(h)$ of $Y(\tilde{j} h)$. This proves the claim.

Assuming $\tau$ has limited support as above, we now define

$$
\begin{gathered}
\Phi_{0}^{n}(\tilde{j} h)=\frac{1}{L} \sum_{\ell=1}^{L / h} \tau(\tilde{j} h, \ell h, n k) h, \\
\Phi_{d}^{n}\left(\tilde{j} h, j_{d} h\right)=\sum_{\ell=1}^{j_{\ell}}\left(\tau(\tilde{j} h, \ell h, n k)-\Phi_{0}^{n}(\tilde{j} h)\right) h
\end{gathered}
$$

for $1 \leq j_{d} \leq L / h$ and $\Phi_{d}^{n}(\tilde{j} h, 0)=0$. $\Phi_{0}^{n}$ extends to a periodic function since it depends only on $\tilde{j} h$, and $\Phi_{d}^{n}$ extends periodically since $\Phi_{d}^{n}(\tilde{j} h, L)=0$. Moreover

$$
\tau=D_{d}^{-} \Phi_{d}^{n}+\Phi_{0}^{n}
$$


From the claim above, there are only $O(1)$ nonzero terms in (8.17) and thus $\left\|\Phi_{0}^{n}\right\| \leq$ $C h\|\tau\| \leq C^{\prime} h^{2}$. Then for the same reason $\left\|\Phi_{d}^{n}\right\| \leq C_{1} h\|\tau\|+C_{2} h^{2} \leq C^{\prime} h^{2}$. This proves the lemma for the special case we have considered.

For general $\tau$, we can use compactness to cover the time-dependent boundary by a finite number of coordinate patches in which one spatial coordinate is a function of the others. The irregular points for each such patch can be handled as above. (Cf. the proof of Lemma 2.2 in [3].) For the remaining regular points, $\tau=O\left(h^{2}\right)$, and these points contribute to $\Phi_{0}^{n}$.

Appendix A. Particular Schemes. We summarize the four time-stepping methods that are emphasized here, as applied to (1.1). All are second-order accurate and A-stable. All except CN are L-stable. We give the function $s(z)$ for the single-step methods.

The familiar Crank-Nicolson $(\mathrm{CN})$ method is

$$
\begin{gathered}
\left(1-\frac{k}{2} \Delta_{h}\right) u^{n+1}=\left(1+\frac{k}{2} \Delta_{h}\right) u^{n}+k f^{n+1 / 2}, \\
s(z)=(1+z / 2)(1-z / 2)^{-1} .
\end{gathered}
$$

The TGA method, as defined in [25] and adapted in [18], is

$$
\left(1-k \mu_{1} \Delta_{h}\right)\left(1-k \mu_{2} \Delta_{h}\right) u^{n+1}=\left(1+k \mu_{3} \Delta_{h}\right) u^{n}+\left(1+k \mu_{4} \Delta_{h}\right) k f^{n+1 / 2}
$$

where $a$ is chosen with $1 / 2<a<2-\sqrt{2}$ and

$$
\begin{gathered}
\mu_{1}, \mu_{2}=\left(a \mp \sqrt{a^{2}-4 a+2}\right) / 2, \quad \mu_{3}=1-a, \quad \mu_{4}=(1 / 2)-a \\
s(z)=\left(1+\mu_{3} z\right)\left(1-\mu_{1} z\right)^{-1}\left(1-\mu_{2} z\right)^{-1} .
\end{gathered}
$$

SDIRK2, a singly diagonally implicit Runge-Kutta method, is (e.g., see [8], p. 98)

$$
\begin{gathered}
u^{n+1}=u^{n}+\frac{k}{2}\left(K_{1}+K_{2}\right), \\
K_{1}=\Delta_{h} u^{n}+k \gamma \Delta_{h} K_{1}+f^{n+\gamma} \\
K_{2}=\Delta_{h} u^{n}+k(1-2 \gamma) \Delta_{h} K_{1}+k \gamma \Delta_{h} K_{2}+f^{n+1-\gamma}
\end{gathered}
$$

where

$$
\begin{gathered}
\gamma=(2 \pm \sqrt{2}) / 2 \\
s(z)=(1+(1-2 \gamma) z)(1-\gamma z)^{-2} .
\end{gathered}
$$

BDF2, the second-order backward difference formula is (e.g., see [8], pp. 364-66, or [9], p. 27)

$$
\frac{3}{2} u^{n+1}-2 u^{n}+\frac{1}{2} u^{n-1}=k \Delta_{h} u^{n+1}+k f^{n+1} .
$$

BDF2 is discussed further in Sec. 6 .

\section{REFERENCES}

[1] A. Ashyralyev and P. E. Sobolevskii, Well-posedness of parabolic difference equations, Birkhäuser, Basel, 1994. 
[2] N. Bakaev, V. Thomée, and L. Wahlbin, Maximum-norm estimates for resolvents of elliptic finite element operators, Math. Comp. 72 (2003), 1597-1610.

[3] J. T. Beale and A. Layton, On the accuracy of finite difference methods for elliptic problems with interfaces, Commun. Appl. Math. Comput. Sci. 1 (2006), 91-119 (www.camcos.org).

[4] N. Dunford and J. Schwartz, Linear Operators: Part I, Interscience, New York, 1958.

[5] R. Glowinski, Finite element methods for incompressible viscous flow, Handbook of Numerical Analysis, Vol. IX, North-Holland, 2003.

[6] B. Griffith and C. Peskin, On the order of accuracy of the immersed boundary method: Higher order convergence rates for sufficiently smooth problems, J. Comput. Phys. 208 (2005), 75-105.

[7] E. Hairer, S. Norsett and G. Wanner, Solving Ordinary Differential Dquations I, Nonstiff Problems, second ed., Springer, Berlin, 1993.

[8] E. Hairer and G. Wanner, Solving Ordinary Differential Dquations II, Stiff and Differential Algebraic Problems, second ed., Springer, Berlin, 1996.

[9] A. Iserles, A First Course in the Numerical Analysis of Differential Equations, Cambridge Press, Cambridge, 1996.

[10] F. John, On the integration of parabolic equations by difference methods, I: Linear and quasilinear equations for the infinite interval, Comm. Pure Appl. Math. 5 (1952), 155-211.

[11] L. Lee and R. LeVeque, An immersed interface method for incompressible Navier-Stokes equations, SIAM J. Sci. Comput. 25 (2003), 832-856.

[12] R. J. LeVeque and Z. Li, The immersed interface method for elliptic equations with discontinuous coefficients and singular sources, SIAM J. Numer. Anal. 31 (1994), 1019-1044.

[13] Z. Li and K. Ito, The Immersed Interface Method, SIAM, Philadelphia, 2006.

[14] Z. Li and K. Ito, Maximum principle preserving schemes for interface problems with discontinuous coefficients, SIAM J. Sci. Comput. 23 (2001), 339-361.

[15] Z. Li and M.-C. Lai, The immersed interface method for the Navier-Stokes equations with singular forces, J. Comput. Phys. 171 (2001), 822-42.

[16] M. Luskin and R. Rannacher, On the smoothing property of the Crank-Nicolson scheme, Applicable Anal. 14 (1982/83), 117-35.

[17] A. Mayo, The fast solution of Poisson's and the biharmonic equations on irregular regions, SIAM J. Numer. Anal. 21 (1984), 285-299.

[18] P. McCorquodale, P. Colella and H. Johansen, A Cartesian grid embedded boundary method for the heat equation on irregular domains, J. Comput. Phys. 173 (2001), 620-35.

[19] S. Nordmark, Uniform stability of a class of parabolic difference operators, BIT 14 (1974), 314-25.

[20] R. Richtmyer and K. Morton, Difference Methods for Initial-Value Problems, second ed., Interscience, 1967.

[21] V. Rutka, Immersed Interface Methods for Elliptic Boundary Value Problems, dissertation, T. U. Kaiserslautern, 2005.

[22] V. Thomée, Finite difference methods for linear parabolic equations, Handbook of Numerical Analysis, Vol. I, North-Holland, Amsterdam, 1990.

[23] V. Thomée, Galerkin Finite Element Methods for Parabolic Problems, second ed., Springer, Berlin, 2006.

[24] J. Trangenstein and C. Kim, Operator splitting and adaptive mesh refinement for the Luo-Rudy I model, J. Comput. Phys. 196 (2004), 645-79.

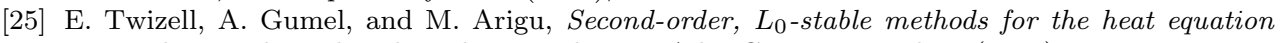
with time-dependent boundary conditions, Adv. Comput. Math. 6 (1996), 333-52.

[26] O. Widlund, Stability of parabolic difference schemes in the maximum norm, Numer. Math. 8 (1966), 186-202.

[27] D. Xiu and G. Karniadakis, A semi-Lagrangian high-order method for Navier-Stokes equations, J. Comput. Phys. 172 (2001), 658-84. 